\title{
Latest Results on Bottom Spectroscopy and Production with CDF
}

\author{
Igor V. Gorelov* \\ Department of Physics and Astronomy, \\ MSC07 4220, University of New Mexico, \\ 800 Yale Blvd. NE, \\ Albuquerque, NM 87131, USA
}

\begin{abstract}
Using data collected with the CDF Run II detector, new measurements on bottom production cross-sections are presented. The latest achievements in bottom hadron spectroscopy are discussed. The results are based on a large sample of semileptonic and hadronic decays of bottom states made available by triggers based on the precise CDF tracking system.
\end{abstract}

\section{First Observation of the Baryons $\Sigma_{\mathrm{b}}$ and $\Sigma_{\mathrm{b}}^{*}$ in CDF}

The bottom $\Sigma_{b}^{(*)}$ states decay strongly into $\Lambda_{b}^{0}$ by emitting soft pion as shown in Figure 1 . Our results are based on data collected with the CDF II detector [2] and corresponding to an integrated luminosity of $\sim 1.1 \mathrm{fb}^{-1}$. The trigger used in this study is based on displaced tracks. It reconstructs with the central tracker a pair of $p_{T} \gtrsim 2.0 \mathrm{GeV} / c$ tracks at Level 1 and enables secondary vertex selection at Level 2 requiring each of these tracks to have impact parameter measured by the CDF silicon detector SVX II larger than $120 \mu \mathrm{m}$. The signals of $\Sigma_{b}^{(*) \pm}$ states were sought in the decay chain $\Sigma_{b}^{(*) \pm} \rightarrow \Lambda_{b}^{0} \pi_{\text {soft }}^{ \pm}$, $\Lambda_{b}^{0} \rightarrow \Lambda_{c}^{+} \pi^{-}, \Lambda_{c}^{+} \rightarrow p K^{-} \pi^{+\mathrm{a}}$. To remove the contribution due to a mass resolution

*This talk [1] has been presented on behalf of the CDF Collaboration at a conference "Photon 2007".

${ }^{a}$ Unless otherwise stated all references to the specific charge combination imply the charge conjugate combination as well.

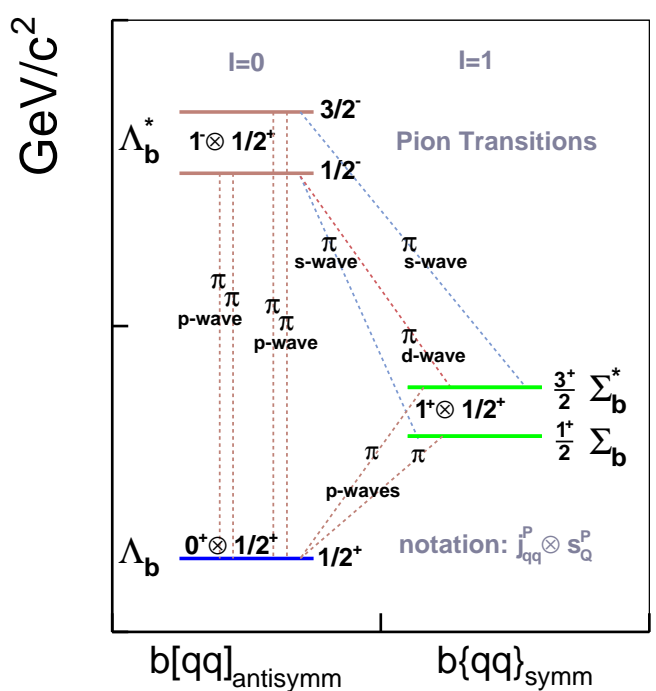

Figure 1: The low lying $\Sigma$ - and $\Lambda$ - like $b$ baryons and their strong decays with pion emissions.

of each $\Lambda_{b}^{0}$ candidate and to avoid absolute mass scale systematic uncertainties, the $\Sigma_{b}^{(*) \pm}$ candidates were reconstructed in the mass difference $Q$-value spectra defined as $Q=M\left(\Lambda_{b}^{0} \pi_{\text {soft }}^{ \pm}\right)-M\left(\Lambda_{b}^{0}\right)-M_{\mathrm{PDG}}\left(\pi^{ \pm}\right)$for every charge state of $\Sigma_{b}^{(*) \pm}$ candidates. Here we assume also that the width of the weakly decaying $\Lambda_{b}^{0}$ candidate is determined by the corresponding detector mass resolution. The fitted experimental spectra are shown at Figure 2, and fit results are summarized in Tables 1 and 2 [3]. 


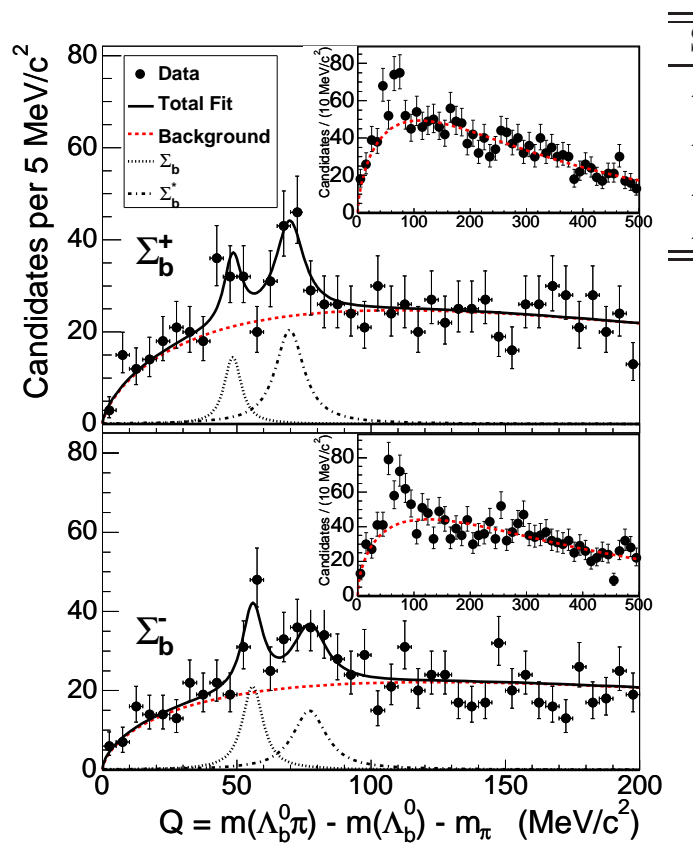

Figure 2: The experimental mass difference spectra [3] for the candidates of both charged partners, $\Sigma_{b}^{(*) \pm}$. Double peak signatures are observed in every case.

\section{Observation and Mass Mea- surement of the Baryon $\Xi_{\mathrm{b}}$}

The bottom cascade baryons $\Xi_{b}$ consist of a single bottom quark, one strange quark and one light quark. Theoretical predictions for these heavy baryons are outlined in Table 3 [4]. We consider the lowest lying $\Xi_{b}$ states that decay weakly and the $\Xi_{b}^{\prime}$ states that decay radiatively or strongly via pion emission. The $\Xi_{b}$ candidates are reconstructed in the decay chain $\Xi_{b}^{-} \rightarrow J / \psi \Xi^{-}$ with secondary states $J / \psi \rightarrow \mu^{+} \mu^{-}$and $\Xi^{-} \rightarrow \Lambda^{0} \pi^{-}, \Lambda^{0} \rightarrow p \pi^{-}$(see Figure 3 ) - Since experiments with bubble chambers the strange cascade, given its long decay path of $c \cdot \tau=4.91 \mathrm{~cm} \mathrm{[5],} \mathrm{is} \mathrm{identified} \mathrm{as}$ a charged track with a 1-track decay vertex at the end formed by a kinked soft pion track as shown at Figure 3. The subse-

\begin{tabular}{lll}
\hline State & \multicolumn{1}{c}{$Q$ or $\Delta_{\Sigma_{b}^{*}}\left(\mathrm{MeV} / c^{2}\right)$} & \multicolumn{1}{c}{ Mass $\left(\mathrm{MeV} / c^{2}\right)$} \\
\hline$\Sigma_{b}^{+}$ & $Q_{\Sigma_{b}^{+}}=48.5_{-2.2-0.3}^{+2.0+0.2}$ & $5807.8_{-2.2}^{+2.0} \pm 1.7$ \\
$\Sigma_{b}^{-}$ & $Q_{\Sigma_{b}^{-}}=55.9 \pm 1.0 \pm 0.2$ & $5815.2 \pm 1.0 \pm 1.7$ \\
$\Sigma_{b}^{*+}$ & $\Delta_{\Sigma_{b}^{*}}=21.2_{-1.9-0.3}^{+2.0+0.4}$ & $5829.0_{-1.8-1.8}^{+1.6+1.7}$ \\
$\Sigma_{b}^{*-}$ & $5836.4 \pm 2.0_{-1.7}^{+1.8}$ \\
\hline
\end{tabular}

Table 1: The masses resulting from the simultaneous fit of both spectra [3].

\begin{tabular}{cccc}
\hline \hline \multicolumn{4}{c}{ Yields of the signals } \\
\hline$\Sigma_{b}^{+}$ & $\Sigma_{b}^{-}$ & $\Sigma_{b}^{*+}$ & $\Sigma_{b}^{*-}$ \\
\hline $32_{-12-3}^{+13+5}$ & $59_{-14-4}^{+15+9}$ & $77_{-16-6}^{+17+10}$ & $69_{-17-5}^{+18+16}$ \\
\hline \hline
\end{tabular}

Table 2: The fitted yields [3] of the identified $\Sigma_{b}^{(*) \pm}$ states. The combined significance of all four peaks relative to the null hypothesis well exceeds 5 Gaussian standard deviations.

quent $V^{0}$ decay vertex of the $\Lambda^{0}$ is associated with the 1 -track vertex and included in a two-vertex kinematic fit. The key technique in this analysis is the tracking algorithm developed to reconstruct $\Xi^{-}$tracks leaving hits in the CDF silicon vertex tracker SVX II. A finest tracking resolution [2] coupled with the custom software provide a clean signal for $\Xi^{-}$, see Figure 4 . The analysis [6] uses a data sample of integrated $\mathcal{L}=$ $1.9 \mathrm{fb}^{-1}$ collected by the CDF dimuon trigger [2] which saves events with two oppositely charged tracks reconstructed in the CDF cen-

\begin{tabular}{llllll}
\hline \hline State & $b s q$ & $J^{P}$ & $I_{3}$ & $j_{s q}$ & $\mathrm{M}, \mathrm{GeV} / c^{2}$ \\
\hline$\Xi_{b}^{0}$ & $b[s u]$ & $1 / 2^{+}$ & $1 / 2$ & 0 & 5.80 \\
$\Xi_{b}^{-}$ & $b[s d]$ & $1 / 2^{+}$ & $-1 / 2$ & 0 & 5.80 \\
$\Xi_{b}^{0^{\prime}}$ & $b\{s u\}$ & $1 / 2^{+}$ & $1 / 2$ & 1 & 5.94 \\
$\Xi_{b}^{--^{\prime}}$ & $b\{s d\}$ & $1 / 2^{+}$ & $-1 / 2$ & 1 & 5.94 \\
\hline \hline
\end{tabular}

Table 3: Theoretical expectations for properties of bottom cascade baryons containing a single $b$ - quark [4]. The lowest lying states have a light quark pair with momentum $j_{s q}=0$ while the next ones have light quarks aligned with $j_{s q}=1$. 


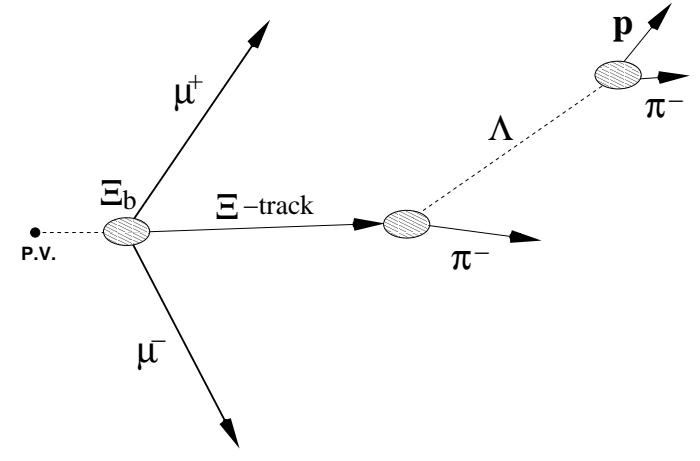

Figure 3: Topology of the $\Xi_{b}^{-} \rightarrow J / \psi \Xi^{-}$decay.

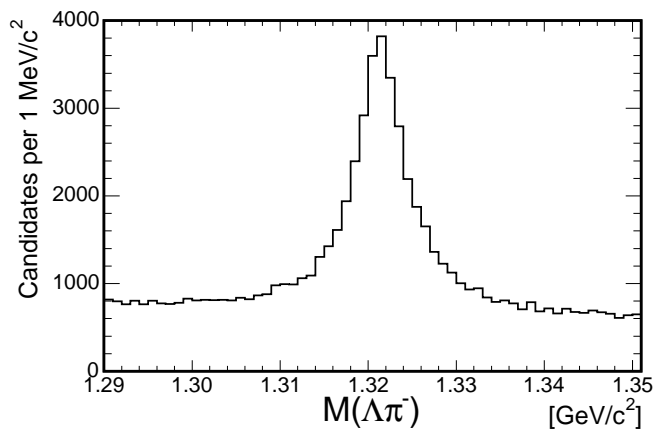

Figure 4: The $\Xi^{-}$signal [6] when the cascade track has at least 2 hits in the CDF SVX II tracker.

tral tracker, matched to hits in the CDF muon chambers and selected in the mass window $M\left(\mu^{+} \mu^{-}\right) \in[2.7,4.0] \mathrm{GeV} / c^{2}$ around the mass of the $J / \psi[5]$. The sample yields $\sim 15 \times 10^{6} \mathrm{~J} / \psi$ and $\sim 23500 \Xi^{-}$candidates. The final selection criteria for $\Xi_{b}^{-}$ candidates have been studied using $\sim 31000$ $B$-mesons in the mode $B^{+} \rightarrow J / \psi K^{+}$as a control sample assuming very similar decay kinematics. The invariant mass of selected $J / \psi \Xi^{-}$candidates is shown in Figure 5. An unbinned likelihood fit finds [6] $17.5 \pm 4.3$ (stat) $\Xi_{b}$ candidates at a mass of $5792.9 \pm 2.5$ (stat) \pm 1.7 (syst) $\mathrm{MeV} / \mathrm{c}^{2}$ and with a significance of 7.7 of Gaussian standard de-

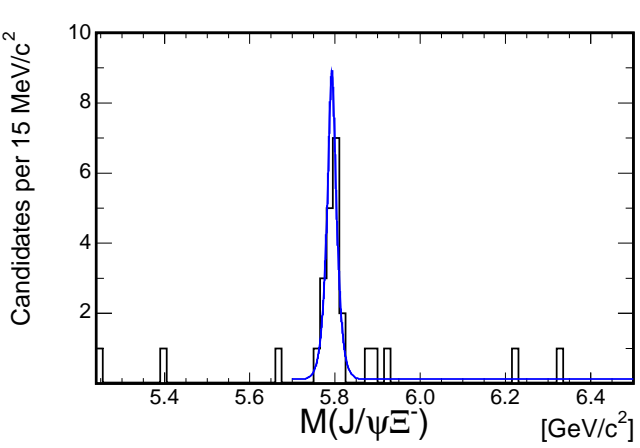

Figure 5: The invariant mass distribution of $J / \psi \Xi^{-}$candidates after optimized selection criteria have been applied. The profile of the unbinned fit is superimposed. A clear signal is observed [6].

viations. The results [6] are in good agreement with theoretical predictions and with the observation made by the $\mathrm{D} \varnothing$ Collaboration [7].

\section{Correlated $b \bar{b}$ Production in CDF II Detector}

In this chapter we cover briefly a unique analysis on a paired $b \bar{b}$ production measurement. As leading order (LO) processes dominate $b \bar{b}$ production, $\sigma_{b \bar{b}}$, while next-to-leading (NLO) processes are essential for inclusive $\sigma_{b}$ studies, the measurement of $\sigma_{b \bar{b}}$ will help to disentangle LO and NLO contributions and to resolve the controversy between the Run I D $\varnothing$ and CDF measurements [8]. We select dimuon events with invariant masses $5<M\left(\mu_{1} \mu_{2}\right)<80 \mathrm{GeV} / c^{2}$, outside of the domain populated by sequential decays of single $b$ - quarks and $Z^{0}$ modes, and extract $\sigma\left(b \rightarrow \mu^{-}+X, \bar{b} \rightarrow \mu^{+}+X\right)$, subtracting contributions from $c \bar{c}$, prompt Drell-Yan pairs, $c$ - and $b$ - onium prompt decays, $\pi$-, $K$ decays, and misidentified dimuon candidates. The signal and background contributions are determined by fitting the experimental 2dimensional impact parameter $d_{0}\left(\mu_{1}\right), d_{0}\left(\mu_{2}\right)$ 
distribution to corresponding templates expected for various dimuon sources. The method exploits the fact that the shape of the $d_{0}(\mu)$ distribution is largely determined by the lifetime of its parent heavy hadron. The analysis is based on a data sample of total luminosity $\mathcal{L}=740 \mathrm{pb}^{-1}$ collected with the CDF dimuon trigger [2] having no biases with respect to $d_{0}(\mu)$ distribution. The projection of the 2 -dimensional fit onto $d_{0}(\mu)$ comprising various background contributions is shown in Figure 6. The extracted exper-
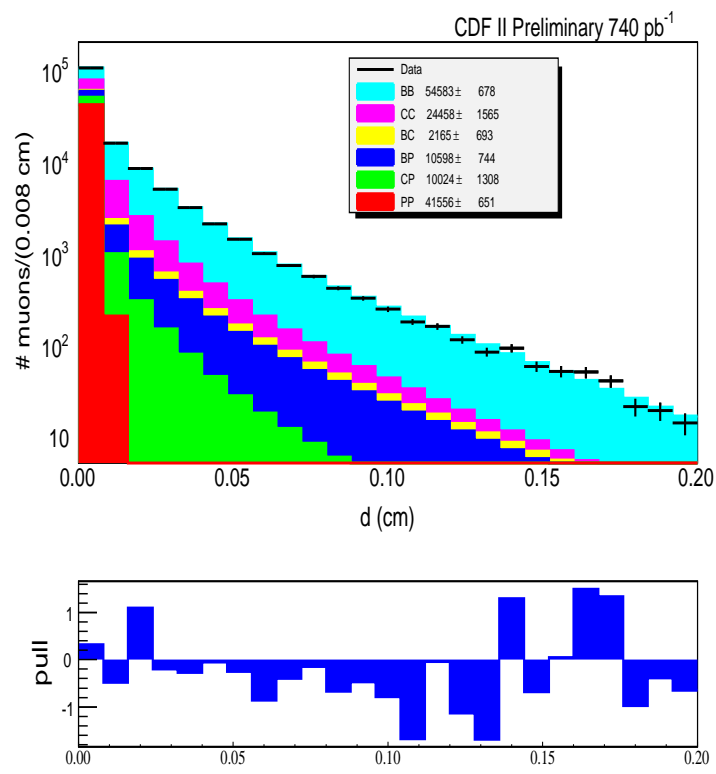

Figure 6: The projection of the 2dimensional fit of $d_{0}\left(\mu_{1}\right), d_{0}\left(\mu_{2}\right)$ with background templates summed up and data superimposed. The notations used are "B" as b-source, "C" as $c$-source and "P" as the source of prompt muons.

imental cross-section is found to be $\sigma(b \rightarrow$ $\left.\mu^{-}, \bar{b} \rightarrow \mu^{+}\right)=1549 \pm 133 \mathrm{pb}$. The exact NLO predictions are made using Herwig Monte-Carlo program [9], MNR code [10] running with EVTGEN generator [11], parton structure functions from MRST [12] fits and Peterson fragmentation function [13].
The ratio of data to NLO theoretical MonteCarlo calculation is found to be $R 2(b \rightarrow$ $\left.\mu^{-}, \bar{b} \rightarrow \mu^{+}\right)=1.20 \pm 0.21$. The errors include statistical and systematic uncertainties added in quadratures. From this measurement we derive $\sigma\left(b \bar{b}, p_{T} \geq 6 \mathrm{GeV} / c,|y| \leq\right.$ $1)=1618 \pm 148 \mathrm{nb}$. The systematic uncertainty due to choice of the fragmentation model is $\sim 25 \%$.

\section{Summary}

CDF announces the first observation of four bottom baryon $\Sigma_{b}^{(*) \pm}$ resonance states. CDF has also observed the strange bottom cascade baryon $\Xi_{b}^{-}$, and our measurements are in agreement with the $\mathrm{D} \varnothing$ observation and with theoretical predictions. CDF II detector has measured the correlated production cross-section of $b \bar{b}$ pairs with $b$-quarks identified in their muonic semileptonic modes. The measurement is consistent with theoretical expectations. Using NLO Monte-Carlo cross-section calculations, the full $b \bar{b}$ production cross-section in the kinematic domain $\left(p_{T} \geq 6 \mathrm{GeV} / c,|y| \leq 1\right)$ has been derived.

\section{Acknowledgments}

The author is grateful to his colleagues from the CDF B-Physics Working Group for useful suggestions and comments made during preparation of this talk. The author thanks S. C. Seidel for support of this work.

\section{References}

[1] Slides: http://indico.cern.ch/materialDisplay.py? contribId $=53 \&$ session $I d=18$ \&material Id=slides\&conf $I d=3841$

[2] D. Acosta et al. (CDF Collaboration), Phys. Rev. D 71032001 (2005).

[3] T. Aaltonen et al. (CDF Collaboration), arXiv:0706.3868v1 [hep-ex]. Submitted to Phys. Rev. Lett. .

[4] J. G. Körner, M Krämer and D. Pirjol, Prog. Part. Nucl. Phys. 33787 (1994), [arXiv:hep$\mathrm{ph} / 9406359 \mathrm{v} 1]$ and references herein. 
[5] W-M Yao et al. (Particle Data Group) J. Phys. G 331 (2006).

[6] T. Aaltonen et al. (CDF Collaboration), Phys. Rev. Lett. 99052002 (2007), [arXiv:0707.0589v2 [hep-ex]].

[7] V. M. Abazov et al. (The D $\varnothing$ Collaboration), Phys. Rev. Lett. 99052001 (2007), [arXiv:0706.1690v3 [hep-ex]].

[8] F. Happacher, P. Giromini and F. Ptohos, "Status of the observed and predicted $b \bar{b}$ production at the Fermilab Tevatron ", Phys. Rev. D 73 014026 (2006). See also references herein.

[9] S. Frixione, P. Nason and B. R. Webber, JHEP 0308, 007 (2003) [arXiv:hep-ph/0305252].

See also

//http:/www.hep.phy.cam.ac.uk/theory/webber/MCatNLO for code downloads.

[10] M. L. Mangano, P. Nason and G. Ridolfi, Nucl. Phys. B 373, 295 (1992).

See also //http:/www.ge.infn.it/ridolti for code downloads.

[11] D. J. Lange, Nucl. Instrum. Meth. A 462, 152 (2001).

[12] A. D. Martin, R. G. Roberts, W. J. Stirling and R. S. Thorne, Eur. Phys. J. C 4, 463 (1998) [arXiv:hep-ph/9803445].

[13] C. Peterson et al., Phys. Rev. D 27105 (1983). 